EPJ Web of Conferences 71, 00040 (2014)

DOI: $10.1051 /$ epjconf / 20147100040

(C) Owned by the authors, published by EDP Sciences, 2014

\title{
Three P's in Cosmology: Progress, Problems, and Perspectives
}

\author{
A.D. Dolgov ${ }^{1-4, a}$ \\ ${ }^{1}$ Novosibirsk State University, Novosibirsk, 630090, Russia \\ ${ }^{2}$ ITEP, Bol. Cheremushkinsaya ul., 25, 113259 Moscow, Russia \\ ${ }^{3}$ Dipartimento di Fisica e Scienze della Terra, Università degli Studi di Ferrara \\ Polo Scientifico e Tecnologico - Edificio C, Via Saragat 1, 44122 Ferrara, Italy \\ ${ }^{4}$ Istituto Nazionale di Fisica Nucleare (INFN), Sezione di Ferrara \\ Polo Scientifico e Tecnologico - Edificio C, Via Saragat 1, 44122 Ferrara, Italy
}

\begin{abstract}
A review on the development of cosmology for a diverse audience is presented. The first historical part is devoted to the works done half a century ago with an emphasize to those performed in Russia (or more precisely, in the Soviet Union). Then the recent data on the cosmic microwave background raditation is discussed and the impact of these data on the neutrino mass and the number of neutrino species is considered. The latter is compared with the results obtained from the big bang nucleosynthesis. Next, a short description and history of the cosmological inflation is presented. The basics of the standard cosmological model and the problems of dark matter and dark energy are considered at the end of the fist section entitled "Progress". In the next short section, "Problems", one can find a list of the unsolved cosmological and astrophysical problems, which is by no means fully comprehensive. The last supershort section contains a few words about the perspectives of future development.
\end{abstract}

\section{Progress}

\subsection{Historical introduction}

It is difficult to cover the impressive development of cosmology of the last half century in half an hour, so my presentation surely misses some essential contributions and in addition may be strongly biased towards Russian papers (and inside that to works done in my home institute, ITEP), some of which are not well appreciated outside Russia.

In the sixties the general attitude to cosmology from the "fundamental" physics establishment was well characterized by the sarcastic quotation from L.D. Landau describing it as a science which is "always in error but never in doubt." Still even at that "dark times" many great works have been done building the cornerstones of the modern cosmology. Of course all contemporary cosmology originated from the Einstein's General Relativity [1] and from the Friedmann cosmological solution of the Einstein's equations [2], where the universe expansion was predicted. Later it was triumphantly confirmed by the Le Maitre [3] and Hubble [4] discoveries. And surely the name of George Gamow, the father of Big Bang cosmology, should be mentioned. In particular from his and collaborator's

\footnotetext{
ae-mail: dolgov@fe.infn.it
} 
works followed an existence of the cosmic microwave background (CMB) radiation [5], whose discovery [6] almost 20 years after the seminal Gamow's papers put to an end the dispute between the supporters of hot versus cold universe models.

Returning to the more recent era inside a half a century interval from today, it is worth mentioning two papers by Zeldovich [7] and Zeldovich, Okun, and Pikelner [8], where the so called freezing of species was first studied. In these works the celebrated equation, whose solution determines the cosmological evolution of the particle density, has been derived. Twelve years later this equation was applied to the calculation of the energy density of stable massive leptons in two very similar papers $[9,10]$ and became known as the Lee-Weinberg equation, which should be surely changed to the Zeldovich equation.

One of the first (if not just the first) suggestions that in addition to the usual baryonic matter there may be some dark matter in the universe (namely mirror matter) was proposed by Kobzarev, Okun, and Pomeranchuk [11].

At the present time big attention is devoted to astronomical methods to put a limit on neutrino mass or even to measure it. Now telescopes are the most sensitive scales to weigh neutrinos (see below, sec. 1.3. All these "measurements" are based on the pioneer paper by Gershtein and Zeldivich [12], where the cosmological number density of neutrinos in the contemporary universe has been calculated. Comparing the energy density of massive neutrinos with the total cosmological energy density the authors were able to derive the bound $m_{v}<30 \mathrm{eV}$. Six years later Cowsick and McClelland [13], using similar considerations, derived noticeably stronger limit but this was because they overestimated the present day cosmological number density of neutrinos by factor 7. A discussion of these problems can be found in the review [14]. So this bound on the neutrino mass would be proper to name the Gerstein-Zeldovich bound.

A year later after the Gerstein-Zeldovich paper Sakharov [15] proposed a beautiful solution to the problem of the baryon asymmetry of the universe, i.e. of the cosmological excess of particles over antiparticles. The Sakharov's suggestion is based on the following three natural conditions: 1) breaking of $\mathrm{C}$ and $\mathrm{CP}$ symmetries; 2) nonconservation of the baryonic number; 3) deviation from thermal equilibrium in the early universe. When Sakharov presented his work, nobody believed that baryonic number may be nonconserved. The motto was: "we exist, ergo baryons are conserved". The today's attitude is exactly opposite: "our existence proves that baryonic number is not conserved". This is because the suitable for life universe can be created, as far as we know, only as a result of inflationary epoch and the latter is impossible with conserved baryons, see sec. 1.4. So nonconservation of baryons can be considered now as an experimental fact proved by astronomy.

Till 1965 most cosmologists in Soviet Union believed that the cold universe model was valid, which was partly explained by the dominant influence of Zeldovich, who liked cold universe. The attitude changed only after the famous Penzias and Wilson discovery of the Cosmic Microwave background (CMB) [16]. Still a half a year before this discovery a seminal paper by Doroshkevich and Novikov [17] was published, where a possibility of registration of CMB and the frequency range most favorable for that, were indicated. It is interesting that 8 years before Penzias and Wilson a similar observation was made by Ter Shamonov [18], who did an analogous work, to that of Penzias and Wilson, on calibration of antenna for a radio telescope. In his case it was the huge Russian RATAN600. However, since the Big Bang cosmology was not popular in Soviet Union at that time, the Ter Shmaonov's result did not attract proper attention and was considered as noise of unknown origin.

Till mid-seventies it was commonly believed that the matter in the universe was predominantly the well known baryonic matter (that is matter consisting of protons, neutrons, and electrons). One has to admit that there were serious reasons for that: we did not observe any noticeable amount of new kind matter in the Earth and, what's more, the spectrum of stellar radiation was that of the normal 
atoms. On the other hand, there already existed astronomical data $[19,20]$ which indicated that there must be some invisible matter in the universe to make the observed velocities compatible with the virial theorem. However, these data were not taken seriously. The breakthrough occurred in 1974 when two groups $[21,22]$ announced that a large part of the cosmological matter is invisible and thus non-baryonic. Now such matter is called generically dark matter (DM) and occupies the central part in publications on astronomy, cosmology, and particle physics.

Development of the observational cosmology can be characterized by comparison of the observational accuracy in the 60 s to that today. The Hubble parameter was known within the factor of two: $H=50-100 \mathrm{~km} / \mathrm{sec} / \mathrm{Mpc}$. The amount of the usual baryonic matter in the universe was determined with the precision of two orders of magnitude: $N_{B} / N_{\gamma}=10^{-9 \pm 1}$, where $N_{B}$ is the average cosmological number density of baryons and $N_{\gamma}$ is the number density of photons in CMS. $95 \%$ of matter in the universe escaped observation: neither dark matter nor dark energy were known. Now the precision is at the per cent level both for the cosmological chemical content and the for the values of the cosmological parameters, see the next section. Cosmology now is an exact science.

\subsection{CMB today}

At the present time the detailed investigation of the frequency spectrum of CMB and of the angular spectrum of its temperature fluctuations became one of the most powerful means to study physics of the early universe and the most precise tool the measure the fundamental cosmological parameters.

The frequency spectrum of CMB is very close to the equilibrium Bose-Einstein spectrum:

$$
f=[\exp ((\omega-\mu) / T)-1]^{-1}
$$

with the temperature $T=2.7260 \pm 0.0013 \mathrm{~K}$ and vanishing chemical potential, $\mu / T<10^{-4}$. The temperature of $\mathrm{CMB}$ radiation coming from different parts in the sky is the same with the relative precision better than $10^{-4}$, excluding the dipole asymmetry $\sim 2 \times 10^{-3}$, which is related to our own motion with respect to the "rest frame" of CMB. However, the perfect isotropy of CMB must be and is distorted due to the inhomogeneous distribution of cosmological matter. The first measurement of the angular temperature fluctuations was performed at the beginning of 90s [23]. Somewhat earlier the results form the Russian satellite Relict were published [24]. However, there only the data on the quadrupole asymmetry were presented and the error bars were quite large: $6.6 \times 10^{-6}<\delta T_{2} / T<$ $3.3 \times 10^{-5}$.

It is interesting to recall that at the time when the detectors for measurement of the CMB fluctuations were under construction, it was expected that the estimated temperature fluctuations should be much larger than those later observed, because the theoretical predictions were made for purely baryonic universe without any dark matter and in this case the amplitude of density should be an order of magnitude larger than in the DM dominated universe (see below sec. 1.6).

Presently there are quite a few precise measurements of the temperature fluctuations of CMB performed on several balloon antennas and satellite WMAP. These results are presented in fig. 1. The analysis of the shape of the presented curve allows to determine the basic cosmological parameters with very high precision. Recently the new very accurate data, especially at small angles, collected by the Planck satellite were published, see fig. 2, which permit to determine cosmological parameters with unprecedented accuracy [25].

The CMB precision in determination of the basic cosmological parameters and the progress made by Planck are illustrated in Table 1 , where $H$ is the Hubble parameter in $\mathrm{km} / \mathrm{sec} / \mathrm{Mpc}, \Omega_{B}, \Omega_{D M}$, and $\Omega_{D E}$ are relative cosmological fraction of baryonic matter, dark matter, and dark energy respectively. These parameters are defined below, eq. (13) 
EPJ Web of Conferences

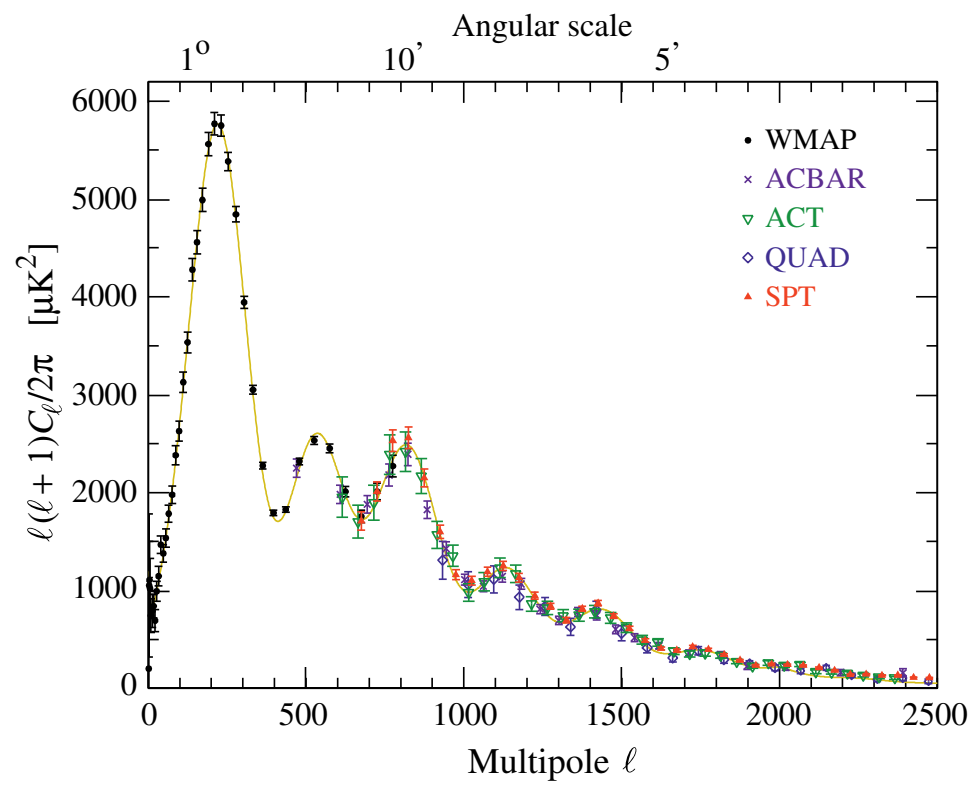

Figure 1. Combined data on the CMB temperature fluctuations before Planck

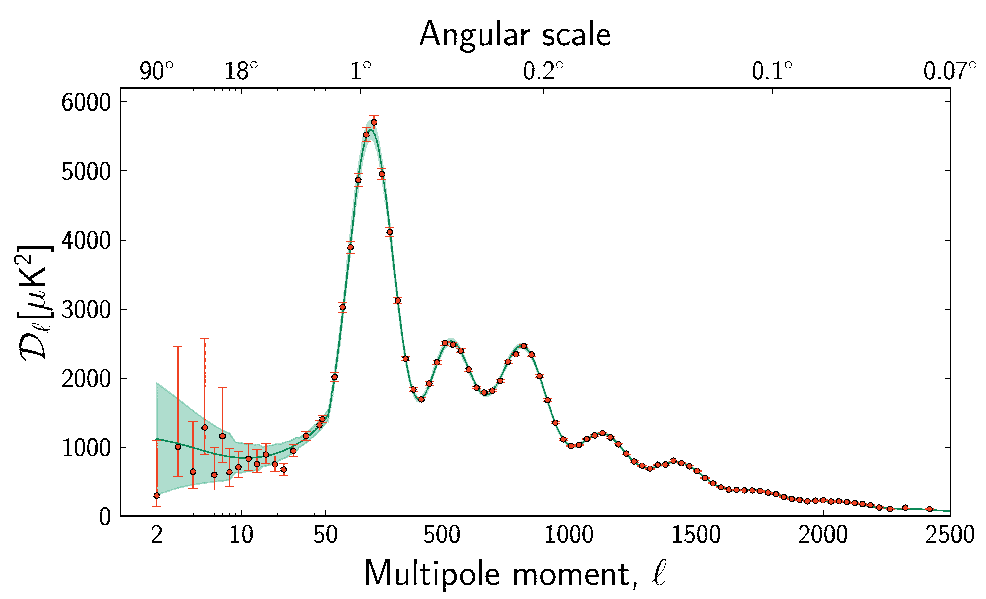

Figure 2. Spectrum of angular fluctuations of CMB measured by Planck

\subsection{Neutrino, CMB, and BBN}

One of the most impressive results from Planck is a very restrictive upper bound on the sum of masses of all three neutrino species::

$$
\sum m_{v}<0.23 \mathrm{eV}
$$


Table 1. Cosmological parameters before and after Planck

\begin{tabular}{lll}
\hline parameter & before Planck & after Planck \\
\hline$H$ & $71 \pm 2.5$ & $67.9 \pm 1.5$ \\
$\Omega_{B}$ & $4.5 \%$ & $4.9 \%$ \\
$\Omega_{D M}$ & $22.7 \%$ & $26.6 \%$ \\
$\Omega_{D E}$ & $72.8 \%$ & $68.3 \%$ \\
\hline
\end{tabular}

Since, as is known from the data on neutrino oscillations, the mass differences among neutrinos are quite small: $\Delta m_{12}^{2} \approx 7.6 \cdot 10^{-5} \mathrm{eV}^{2}$ and $\Delta m_{23}^{2} \approx 2.4 \cdot 10^{-3} \mathrm{eV}^{2}$, the individual neutrino mass is bounded by $m_{v}<0.08 \mathrm{eV}$. which is by far stronger than direct laboratory results on weighing neutrinos. The KATRIN detector, which is under construction, may have comparable sensitivity. It would be especially interesting, if it finds a larger neutrino mass. Such a discovery could lead to a profound modification of the standard cosmological model.

Non-zero $m_{v}$ affects the spectrum of angular fluctuations of CMB in the following way. The position of the peaks would be shifted to the left with rising mass. The larger is $m_{v}$, the earlier is the onset of the non-relativistic cosmological stage. Thus the distance to the last scattering surface becomes shorter and peaks move to smaller $l$. However, one should keep in mind that this shift can be compensated by a shift in $H$ and the complete analysis of the whole data set is necessary. Another effect of the nonzero neutrino mass is a decrease of the peak heights, because with rising $m_{v}$, as we have just mentioned, the matter radiation equality takes place earlier, so the enhancement of the peak amplitude (by the integrated Sachs-Wolfe effect) tends to be weaker,

The analysis of the angular perturbation spectrum allows also to restrict $N_{v}^{(e f f)}$, i.e the number of effective neutrino species in cosmological plasma. This number is defined as the ratio of the real energy density of relativistic particles, without the CMB photons, normalized to the energy density of one massless neutrino plus the same antineutrino species with temperature equal to $(4 / 11)^{1 / 3}=$ 0.7138 of the temperature of the CMB photons. The difference between $T_{v}$ and $T_{\gamma}$ is created by $e^{+} e^{-}$-annihilation which, after neutrino decoupling, heats only the photon gas, while neutrinos remain (almost) untouched. In the standard cosmology with three usual neutrinos $N_{v}^{(e f f)}=3.046$. Naively one would expect $N_{v}^{(e f f)}=3$. Extra 0.011 out of the 0.046 excess comes from the deviation of the $\left(\gamma e^{-} e^{+}\right)$plasma from the ideal state (e.g. from a decrease of the photon energy density due to non-zero plasma frequency) [26] and the remaining 0.035 comes from non-instant neutrino decoupling from $e^{+} e^{-}$pairs, with higher temperature, whose annihilation slightly heats up the neutrino gas and distorts its energy spectrum. This phenomenon was discovered in the work [27] and the first precise calculations were performed in [28], see also review [29] and subsequent papers [30], which confirmed this result. 
According to the Planck data [25] $N_{v}^{(e f f)}=3.30 \pm 0.27$, which does not contradict the canonical value 3.046 but, on the other hand, hints to some extra dark radiation of yet unknown form. Similar results follow from the analysis of big bang nucleosynthesis. The data on the primordial ${ }^{4} \mathrm{He}[31] \mathrm{im}-$ ply for the baryonic density multiplied by the dimensionless Hubble parameter squared, $h=H /(100$ $\mathrm{km} / \mathrm{sec} / \mathrm{mpc})$ :

$$
\Omega_{b} h^{2}=0.0234 \pm 0.0019(68 \% C L)
$$

and for the effective number of the neutrino species:

$$
N_{v}^{(e f f)}=3.51 \pm 0.35(68 \% C L) \text {. }
$$

The result (3) well agrees with that found by Planck:

$$
\Omega_{b} h^{2}=0.02205 \pm 0.00028 .
$$

This agreement is especially impressive because the BBN measurements are sensitive to cosmology of the very early universe with the age $t \sim(1-200) \mathrm{sec}$, while CMB presents information about the universe about 100000 years old.

One more recent determination of $N_{v}^{(e f f)}$ is presented in ref. [32] based on the determination of the primordial deuterium abundance $(D / H)_{p}=(2.53 \pm 0.04) \cdot 10^{-5}$, corresponding to

$$
\Omega_{b} h^{2}=0.02202 \pm 0.00045
$$

for the standard model of BBN. By combining the data on $(D / H)_{p}$ with observations of the cosmic microwave background, the authors of [32] derived for the effective number of neutrinos the value:

$$
N_{v}^{(e f f)}=3.28 \pm 0.28
$$

The presented results for $N_{v}^{(e f f)}$ are compatible with the standard scenario with 3 usual neutrino species. However, it is intriguing that the central values of all measurements are noticeably above 3 . Maybe these data indicate the existence of light sterile neutrino or some other form of dark radiation.

\subsection{Inflation}

A revolutionary development in cosmology took place at the beginning of 80 s when it was understood that an early exponential expansion (inflation) solved in a natural and unique way the "eternal" problems of the Friedmann cosmology: horizon, flatness, homogeneity, creation of the primordial density perturbations, and the origin of expansion. The pioneering papers where the inflationary solution of these problems (except for the density perturbations) belong to D. Kazanas [33] and A. Guth [34].

There are very many different models of inflation now. The most popular one is probably Linde's chaotic inflationary model [35], where exponential expansion is created by a scalar field, $\Phi$, with almost flat potential $U(\Phi)$. In such a potential one can neglect the derivative terms in the energymomentum tensor:

$$
T_{\mu \nu}(\Phi)=\partial_{\mu} \Phi \partial_{\nu} \Phi / 2-(1 / 2) g_{\mu \nu}\left[g^{\alpha \beta} \partial_{\alpha} \Phi \partial_{\beta} \Phi-U(\Phi)\right] \approx(1 / 2) g_{\mu \nu} U(\Phi),
$$

where $g_{\mu \nu}$ is the metric tensor. For such $T_{\mu \nu}$ the equation of state has the form $P \approx-\rho$, where $P$ and $\rho$ are the pressure and energy densities of $\Phi$. Thus the universe would expand exponentially till this equation of state remains valid.

After Planck very precisely measured the spectrum of the primordial density perturbations and found that the power index of the spectrum is $n=0.96$, considerable popularity was gained by the 
Starobinsky $R^{2}$-inflation [36]. This is a cosmological model based on the gravity modification by an addition of $R^{2}$-term to the usual Einstein-Hilbert action: $\sim m_{P l}^{2} R$. In this theory the cosmological expansion would be exponential even in absence of matter. Later it would turn into the Friedmann power law regime after the curvature field decayed into elementary particles.

One of the most important and successful predictions of inflation is the form of the density perturbation spectrum, first calculated by Mukhanov and Chibisov [37], which happens to be very close to the flat $(n=1)$ Harrison-Zeldovich spectrum [38], and quite well agrees with observations.

Another interesting consequence of inflation is a prediction of long relic gravitational waves (GWs) with frequencies roughly in $\mathrm{Hz}$ region [39]. The search for these gravitational waves remains unsuccessful up to now but it does not mean that inflation is excluded, because intensity of the waves depends upon the mechanism of inflation and not only on it but also on the subsequent history of the universe see e.g. [40]. So a registration of such GWs would be a strong additional argument in favor of inflation but their absence would by no means kill inflation.

Anyhow, inflation practically looks as an experimental fact. There is no other way to create suitable for life universe. But beware of "no-go" theorems in physics, so it may be be better to say: we do not see any other way natural to create suitable for life universe. Of course, the anthropic principle may be an alternative, but it is much simpler to implement it demanding the creation of just the Solar system or a small galaxy but not our huge universe. Moreover, the anthropic principle does not predict the shape of the density perturbation spectrum.

It is interesting to note that a sufficiently long inflation is impossible, if baryonic number is conserved [41]. Thus our existence proves that protons or nuclei are unstable. It is amusing that 50 years ago the same "experimental" fact of our existence was used as an argument for the opposite conclusion of baryon conservation. Still direct experimental evidence of proton decay or neutron-antineutron oscillations would be of primary importance.

\subsection{The standard cosmological model}

The reference model in cosmology, the so called the standard cosmological model (SCM) is established. The gross features of the universe history from inflationary creation to the present day is well understood and quantitatively confirmed by the data. However, some "dark clouds" still remain, maybe not as serious as (but maybe much more serious than) the famous two Lord Kelvin's ones: ultraviolet catastrophe and Michelson-Morley problem, out of which the thunderstorms of quantum mechanics and relativity theory broke out. Anyhow there are no doubts about big-bang cosmology and, on the other hand, there are no doubts that cosmology leads us to new physics beyond the minimal standard model of elementary particles and field theory.

\subsubsection{Cosmological equations}

One of the cornerstones of MCM is General Relativity (GR) though it is constantly questioned, especially recently. According to GR the homogeneous and isotropic early universe is described by the the Friedmann equations, which are the special form of the Einstein equations for this particular case. The first Friedmann equation expresses the universe expansion rate, i.e. the Hubble parameter $H$ through the energy density of matter, $\rho$ :

$$
H^{2} \equiv\left(\frac{\dot{a}}{a}\right)^{2}=\frac{8 \pi \rho}{3 m_{P l}^{2}}-\frac{k}{a^{2}},
$$


where $a(t)$ is the cosmological scale factor and $k$ is a constant usually taken as $k= \pm 1$ or $k=0$. The parameter $k$ determines the average spatial geometry of the universe. If $k=1$ the geometry of the 3dimensional space is that of 3-sphere (closed universe), for $k=-1$ it is geometry of the 3 dimensional hyperboloid (open universe), and for $k=0$ the 3 -space is flat with the Euclidian geometry. It was taken for granted that flat and open universes will expand forever, while the closed universe will recollapse back to hot and dense state. It clearly follows from equation (9), if the energy density drops faster than $1 / a^{2}$. After discovery of dark energy whose energy density presumably tends to a constant value, we have to conclude that our universe will expand forever independently of 3-geometry.

The second equation allows to express the cosmological acceleration through the energy, $\rho$, and pressure, $P$, densities of matter:

$$
\frac{\ddot{a}}{a}=-\frac{4 \pi G_{N}}{3}(\rho+3 P)
$$

As follows from this equation in General Relativity (GR), in contrast to Newtonian theory, not only energy but pressure gravitates as well. Life is possible only because of that, since negative pressure, with $(3 P+\rho)<0$, is the source of the cosmological expansion. Note however, that in GR any finite object with positive energy density creates normal attractive gravitational field. Only infinitely large systems can antigravitate. On the other hand, as we see below in sec. 1.7, in infrared modified gravitational theories, which are proposed for an alternative explanation of the observed nowadays accelerated cosmological expansion, finite size systems may exert gravitational repulsion.

The third equation is the law of the covariant conservation of the energy-momentum tensor of matter, $D_{\mu} T_{v}^{\mu}=0$ :

$$
\dot{\rho}+3 H(\rho+P)=0 \text {. }
$$

This equation follows from the first two ones because of the Bianchi identities satisfied by the Einstein tensor: $D_{\mu} R_{v}^{\mu}-\partial_{v} R=0$. So there are only two independent equations for three unknowns, $a(t), \rho(t)$, and $P(t)$. To complete the system we need an equation of state of matter, $P=P(\rho)$, which is usually taken in a simplified linear form

$$
P=w \rho,
$$

where $w$ is supposed to be a constant parameter (but not always); for nonrelativistic matter $w=0$, for relativistic matter $w=1 / 3$, and for vacuum-like matter $w=-1$.

Fractional energy density of different forms of matter, discussed above, is defined as:

$$
\Omega_{j}=\rho_{j} / \rho_{c},
$$

where the critical energy density is

$$
\rho_{c}=\frac{3 H^{2} m_{P l}^{2}}{8 \pi}=0.85 \cdot 10^{-29} \mathrm{~g} / \mathrm{cm}^{3} .
$$

It is equal to the real cosmological energy density if $k=0$ in eq. (9). For $H=67.9 \mathrm{~km} / \mathrm{s} / \mathrm{Mpc}$ the critical energy density has the value:

$$
\rho_{c}=\frac{3 H^{2} m_{P l}^{2}}{8 \pi}=0.85 \cdot 10^{-29} \mathrm{~g} / \mathrm{cm}^{3}=5 \mathrm{keV} / \mathrm{cm}^{3} \approx 4 \cdot 10^{-47} \mathrm{GeV}^{4} \text {. }
$$

The position of the first highest peak in the CMB angular spectrum, figs. 1 and 2, indicates that $\Omega_{t o t}=1$ with a percent precision, so the average spatial geometry of the universe is Euclidean. The arguments go as follows. We know that the physical size of the wave length corresponding to this 
peak is equal to the sound wave horizon at the moment of the hydrogen recombination. The position of this peak corresponds to the angular scale of about 1 degree. Knowing the size of the object and the angle at which the object is observed one can make a conclusion about the geometry of the space where the light propagates. For closed geometry the angle would be larger than for the open one. According to the observations the angle very precisely corresponds to the flat space case.

\subsubsection{Main epochs in the cosmological history}

1. Initial inflationary stage. Exponential expansion of practically empty (with a possible exception for the inflaton field) and dark state. Inflation is practically an experimental fact.

2. Universe heating by the inflaton decay. This process is natural to call Big Bang, when the vacuumlike dark state exploded producing energetic elementary particles. As a result of this explosion the primeval plasma of elementary particles was created closely to thermal equilibrium state. This was, in particular, an origin of cosmic background radiation, which is observed today, being a proof of an early equilibrium and hot state.

3. Baryogenesis i.e. generation of an excess of particles and antiparticles. Inflation proves validity of one of the conditions for baryogenensis, namely of B-nonconservation.

4. Big Bang Nucleosynthesis (BBN). Formation of light primordial elements: ${ }^{2} \mathrm{H}\left(\sim 3 \cdot 10^{-5}\right) ;{ }^{3} \mathrm{He}$ (more or less the same as deuterium); ${ }^{4} \mathrm{He}(\sim 25 \%) ;{ }^{7} \mathrm{Li}\left(\sim 10^{-9}\right)$. The numbers in brackets are relative abundances of the light elements by number, except for ${ }^{4} \mathrm{He}$, for which the mass fraction is presented, relative to the total amount of baryonic matter. For all elements there is a good agreement between theory and observations, except for ${ }^{7} \mathrm{Li}$ for which the observations lie at least by factor 3 below the standard BBN prediction for a discussion see e.g. review [42]

5. Equilibrium epoch, when the energy density of nonrelativistic matter became equal to that of the relativistic one. It takes place at redshift $z_{e q} \approx 10^{4}$, where the cosmological redshift at moment $t$ is defined as $z=a_{0} / a(t)-1$ with $a_{0}$ being the value of the scale factor today. For $z>z_{\text {eq }}$ the universe was mostly dominated by relativistic matter, except for inflation (when $w \approx-1$ ), possible matter dominated stage at the end of inflation, and short periods after possible supercooled first order phase transitions in the primeval plasma. At $z<z_{e q}$ down to $z_{v} \approx 0.7$ the cosmological expansion is determined by nonrelativistic matter and below $z=z_{v}$ the expansion is determined by dark energy with $w \approx-1$.

6. The epoch of large scale structure formation. It started at $z<z_{e q}$, when initially small density fluctuations, which were formed at inflationary stage and remained frozen at $z>z_{e q}$, started to rise as $\delta \rho / \rho \sim a(t)$. This rise continued till $z=z_{v}$, when the fast exponential expansion inhibited a rise of large scale fluctuation.

7. Hydrogen recombination and decoupling of Cosmic Microwave Background radiation (CMB) which took place at $z_{\text {rec }} \approx 1100$. Before recombination the primeval plasma of the usual matter consisted of charged particles, namely of electrons and protons. Due to a large Thomson crosssection electrons were tightly coupled to the photons of CMB, and protons were in turn tightly coupled to electrons through the Coulomb interaction. As a result of that the mean free path of photons was much smaller than the cosmological horizon, $l_{\text {hor }} \sim t$, and the rise of the density perturbations took place in the dark matter sector because the high pressure exerted on electrons by the CMB photons kept them to be homogeneously distributed. After recombination, when the plasma turns into mixture of neutral hydrogen and helium atoms, the structure formation in the sector of the usual matter initiated, when now-neutral matter dropped down into the potential wells already dug by the dark matter. Simultaneously the photons from CMB started to freely propagate over cosmological distances and bring to us today the snapshot of the universe at $z \sim 1100$ which is seen in figs. 1 and 2 . Detectors of CMB are the deepest telescopes which see the universe when it was about $10^{5}$ years old. 
Out of limits of these rather well known periods we enter terra incognita about which we have neither trustable theory not reliable observational data. The first natural direction beyond SCM is an attempt to imagine what happened before inflation, i.e. to formulate the so called pre-big-bang cosmology. Most probably for a description of the universe state quantum gravity at high curvatures would be necessary and correspondingly there would not exist the classical space-time. Maybe multidimensional M-theories could help to proceed in this direction, though it is by no means certain that this is a right way to go. On the other hand, multidimensional theories are interesting independently on pre-big-bang cosmology. There are two different classes of such generalization. Firstly there are theories with small extra dimensions (analogous to the good old Kaluza-Klein theory [43]), and secondly, theories with large extra dimensions, according to which our particles are confined in our 3-dimensional world by some dynamical reason. These kind of theories were suggested rather recently in ref. [44], where it was assumed that we live at three-dimensional domain wall and outside the wall the particle masses are grossly enhanced. Multidimensional theories open rich possibilities for research and hence became very popular at the last decades.

\subsection{Dark matter}

As we have seen above, dark matter (DM) contributes about $26.6 \%$ into the total mass of the universe, at least inside the present day cosmological horizon. DM is an unknown form of otherwise normal matter which obeys the usual laws of gravity. In contrast to electrons, protons. and even neutrons DM does not interact with light and that's why a better name for it could be invisible matter. DM is observed in several different phenomena in the sky due to its gravitational action. Among these phenomena are the following:

1. Flat rotational curves. According to the virial theorem, velocities of any objects bound in gravitational field outside of a confined body drop down as $1 / \sqrt{r}$, where $r$ is the distance to the gravitating center. The measured velocities of gas particles and of galactic satellite around galaxies show surprising "flat" behavior, $r \rightarrow$ const, though no matter is seen outside the luminous center. The natural explanation of this data is that there is some invisible matter around with the mass density which drops down as $\rho \sim 1 / r^{2}$.

2. Gravitational lensing. Light emitted by distant sources is deflected on the way by galaxies and their clusters. The effect is proportional to the total amount of matter, including DM, in these "deflectors". 3. Equilibrium of hot gas in rich galactic clusters. To keep high temperature gas in gravitationally bound state it is necessary to have about 5 times more matter in such clusters than it is directly visible. 4. Cluster evolution. The ratio of the number of galactic clusters today to that in the earlier universe, e.g. at $z \sim 1$, depends upon the total matter density. The smaller is the density of matter, the higher should be this ratio, because the rate of the cluster formation at higher $z$ would be smaller.

5. Combined analysis of LSS (in particular, the baryon acoustic oscillations) and CMB. With known spectrum of the density perturbations the observed today distribution of matter at different scales depends upon the contributions of DM and DE. The same is true for the angular spectrum of CMB.

All these different methods give compatible results $\Omega_{D M} \approx 0.3$. The most precise is the last one.

There is a simple and strong qualitative argument in favor of existence of dark matter, namely, without DM life would not exist at present and possibly never. Indeed, the low measured value of the temperature fluctuations, $\delta T / T$, of $\mathrm{CMB}$, indicates that the density fluctuations at the moment of the hydrogen recombination are also small, of the order of $\delta \rho / \rho \sim 10^{-4}$. As we know, the recombination takes place at $T \approx 3000 \mathrm{~K}$, i.e. $z=1100$, Without DM density perturbations could start rising only after recombination and rise at most as the cosmological scale factor, so today they would remain small, $\delta \rho / \rho<0.1$. Consequently stars and planets would not be created to the present time. They 
could be created much later when the density of matter would drop by three orders of magnitude. It might further inhibit their formation.

From pure astronomical point of view all possible types of DM can be arranged in three classes, depending upon their free streaming length, $l_{F S}$, of DM particles. After these particles decoupled from the cosmological plasma, they freely propagated in the universe till their red-shifted velocity became smaller than their virial velocity. Because of this free streaming density perturbations at the scales below $l_{F S}$ would be evidently erased, since the flux from rich regions to poor ones would be larger than the flux in the opposite direction. The mass of matter inside $l_{F S}$ is called "free streaming mass" and is denoted as $M_{F S}$. Correspondingly:

1. If $M_{F S}>M_{g a l} \sim 10^{12} M_{\odot}$, such matter is called hot dark matter (HDM). An example of the particle of such DM is neutrino with $l_{F S} \sim 0.1 m_{P l}^{3} / m_{v}^{2} \sim 10^{17} M_{\odot}\left(1 \mathrm{eV} / m_{v}\right)^{2}$.

2. If $M_{F S} \sim M_{g a l}$, such DM is called warm (WDM). An example of such DM is given by possible sterile neutrinos with $m \sim \mathrm{keV}$, light pseudogoldstone bosons, or any other keV-mass particles which were in thermal equilibrium in the primeval plasma.

3. If $M_{F S}<M_{\text {gal }}$, the corresponding dark matter is called cold.

The most popular form of DM is the cold one, to large extend because it gives a good gross feature description of LSS and because there are plenty of natural candidates for CDM particles:

1. Till the last year the most popular candidate for CDM was the lightest supersymmetric particle (LSP) with the mass in the interval $m \sim 100-1000 \mathrm{GeV}$. Such particles are stable, if R-parity is conserved, and their calculated cosmological energy density is naturally close to the observed one. However, after no signal from supersymmetry was found at LHS, this possibility significantly lost its popularity. Higher mass LSP can still do the job, but since the cosmological density of LSP is proportional to their mass squared, it would be much larger than the $\rho_{c}$ and some modifications of the standard cosmological scenario would be necessary.

2. Heavy neutral leptons, of the fourth generation $m \sim 2 \mathrm{GeV}$. They may be stable or long-lived if the 4th lepton generation has some conserved quantum number, so that the mixing with the known three generation is absent or very weak. Such or similar particles are generically called WIMPs (weakly interacting massive particles)

3. Ultraheavy, quasi-stable particles (wimpzillas), $m_{X} \sim 10^{13} \mathrm{GeV}$. The problem with such particles is that they should gravitationally decay with the life-time $\tau \sim m_{P l}^{4} / m_{X}^{5} \sim 10^{-12} \mathrm{sec}$. To avoid such a fast decay, one needs to prescribe to X-particles a new conserved gauge quantum number and postulate a new massless gauge boson interacting with it.

4. Axion, $m \sim 10^{-5} \mathrm{eV}$. Despite a very low mass axion could make CDM because it never was in thermal equilibrium and, being produced at rest, was always cold.

5. Primordial black holes with $M>10^{16} \mathrm{~g}$ or some other stellar or planet like dark objects, which got the generic name MACHO's (massive astrophysical compact halo objects).

6. Mirror matter, a whole world of new particles which may be similar to ours, though essential differences are not excluded. Such particles can strongly interact among themselves and dissipate energy into mirror photons, so the structure formation with these particles may be very much different from the canonical case. For a review see ref. [45].

7. Some more exotic possibilities such as non-topological solitons (e.g. Q-balls [46]) or the QCD nuggets [47].

8. The last and maybe the most interesting possibility: none of the above.

In the standard cosmological scenario the so called $\Lambda C D M-c o s m o l o g y$, i.e. a mixture of cold dark matter and dark energy, is assumed. However, CDM has several, possibly serious problems, namely: 1. Missing satellites: CDM predicts an order of magnitude more galactic satellites than observed. 
2. Destruction of galactic disk: even if the number of the satellites is reduced by the star formation winds, many smaller tightly bound DM systems would survive and destroy galactic disk by gravitational heating.

3. Central cusps: expected singularity at galactic centers, $\rho_{D M} \sim r^{-\kappa}, \kappa=1-2$, while flat profiles are observed.

4. Excessive angular momentum: CDM predicts much smaller angular momentum of galaxies than it is observed.

Possible solutions:

1. Insufficient accuracy of numerical simulation or neglected physical effects, e.g. the role of baryons.

2. Dissipative and self-interacting DM (e.g. mirror). However, probably it does not help.

3. WDM, or better, a mixture of WDM and CDM.

\subsection{Dark energy}

Already at the end of the previous century there appeared more and more data indicating that in addition to matter with normal attractive gravitational action the universe is abundantly populated with an antigravitating substance which is similar, in a sense, to that which gave rise to inflation. We know very little about this substance, except for its name: dark energy (DE) and the equation of state (12) with $w=-1.13_{-0.10}^{+0.13}$. It is not even excluded that $w$ may depend upon time.

Antigravity was necessary at very early cosmological stage to create our large suitable for life universe out of microscopically small initial state, but it seems unnecessary now. "Who ordered that?"

Anyhow the existence of dark energy is established beyond any doubts from several independent pieces of the observational data:

a) Universe age crisis, revealed in the previous century. With $H \geq 70 \mathrm{~km} / \mathrm{sec} / \mathrm{Mpc}$ the universe would be too young, $t_{U}<10 \mathrm{Gyr}$, while stellar evolution and nuclear chronology demand $t_{U} \geq 13 \mathrm{Gyr}$.

b) A small value of the cosmological density of the normal matter (including DM), $\Omega_{m}=0.3$, as it was measured by several independent ways, see sec. 1.6. On the other hand, inflation predicts $\Omega_{\text {tot }}=1$ and it is indeed observed through the spectrum of angular fluctuations of CMBR: the position of the first peak "measures" $\Omega_{t o t}=1$.

c) LSS and CMBR very well fit theory if $\Omega_{D E} \approx 0.7$.

d) Dimming of high z supernovae: a certain class of supenova, SN1a, which may be considered as "standard candles", observed at high redshifts, $z \sim 1$, happened to be less bright than expected. It means that they are farther away than they should be if the universe expanded with decreasing speed, as it was believed a quarter of century ago.

The observed supernova dimming cannot be explained by the light absorption on the way because it was found that the effect is non-monotonic as a function of redshift. It reaches maximum at $z \approx 0.7$, as is expected if the dimming is induced by the cosmological acceleration. Indeed, a decrease of the dimming at higher $z$ should take place since the energy density of the usual gravitating matter drops down as $\rho_{m} \sim 1 / a^{3}$, while $\rho_{D E}=$ const and equilibration between gravity and antigravity takes place at $z \approx 0.7$.

The observation of the supenova dimming gained the Nobel Prize of 2011 given to S. Perlmutter, B.P. Schmidt, and A.G. Riess "for the discovery of the accelerating expansion of the Universe through observations of distant supernovae".

Probably the central question about the nature of dark energy is if it coincides with vacuum energy. The energy-momentum tensor of vacuum must be proportional to the only invariant tensor i.e. to the metric tensor:

$$
T_{\mu v}^{(v a c)}=\rho_{v a c} g_{\mu v}
$$


For such tensor $P=-\rho$ and $w=-1$. Observations are compatible with this value but the precision allows for different values as well. It is interesting that $w<-1$ is not excluded. With this negative $w$ we arrive to the so called phantom cosmology, when during a finite time the universe will reach a singular state with infinitely large energy density. The repulsive antigravity created by such a state would be infinitely strong and everything would be turn apart, not only atoms and nuclei but probably even hadrons would be disintegrated into quarks. However this conclusion is based on the analysis of the homogeneous solution of the equations of motion and one may hope that small scale inhomogeneities might save the world from complete destruction.

If $w$ happens to be exactly equal to $(-1)$, then it cannot be formally excluded very dull possibility that dark energy is simply trivial vacuum energy (or, what is the same, just the Lambda-term). On the other hand if vacuum energy is not zero and gravitating, there emerges a fantastic problem of an extreme fine-tuning. There are several natural contributions into $\rho_{v a c}$ which are larger than the observed one by 50-100 orders of magnitude. The most striking of these contribution is that originating from the vacuum condensates of quarks [48] and gluons [49]:

$$
\langle\bar{q} q\rangle_{v a c} \neq 0, \quad\left\langle G_{\mu \nu} G^{\mu v}\right\rangle_{v a c} \neq 0
$$

whose energies are larger than $\rho_{c}$ "only" by 45 orders of magnitude, but still it is not a very small number. In other words the QCD vacuum is not empty but filled with the condensates with negative energy density:

$$
\rho_{\text {vac }}^{Q C D} \approx-10^{45} \rho_{c} .
$$

The necessity of such condensates is evident from the consideration of the value of the proton mass. The proton is known to consist of three quarks with masses about $5 \mathrm{MeV}$ each. Thus one may naively expect that $m_{p}$ should be $15 \mathrm{MeV}$ minus the binding energy, instead of $938 \mathrm{MeV}$. Vacuum condensate is destroyed by quarks, so it is absent inside proton and the proton mass is:

$$
m_{p}=2 m_{u}+m_{d}-\rho_{v a c} l_{p}^{3} \approx 1 \mathrm{GeV},
$$

where $l_{p} \sim 1 / 100 \mathrm{MeV}$ is the proton size.

Quantum chromodynamics (QCD) is well established and experimentally verified science, so the statement about non-zero contribution to vacuum energy from quarks and gluons can be considered as rigorously established fact. On the other hand, the observed vacuum energy density is by far smaller than this QCD contribution. So we have to conclude that something else lives in vacuum and makes the necessary "donation" to compensate $\rho_{\text {vac }}^{Q C D}$ with $10^{-45}$ precision but not to identical zero. In fact this should be not compensation but overcompensation to change the sign of the vacuum energy from negative to positive. This compensating field does not know anything about quarks and gluons, otherwise it would be discovered in experiment. The only natural agent which may eliminate vacuum energy seems to be gravity itself operating through some adjustment mechanism. At the moment no satisfactory mechanism is known and several other possibilities are considered such as a modification of gravity in such a way that $\rho_{v a c}$ does not gravitate, infrared screening of vacuum energy, anthropic principle with almost infinite set of subtraction constants, and maybe much more.

Solution of the DE problem is most probably impossible without understanding of the mechanism of compensation of vacuum energy. However, phenomenological description of DE may be instructive. The problem is aggravated by the cosmic coincidence: why at the present time dark energy makes comparable to the usual matter contribution into the cosmological energy density, though they evolve very differently during cosmological history: $\rho_{D M} \sim 1 / a^{3}$ and $\rho_{D E} \sim$ const.

There are basically two main possibilities for the phenomenological description of dark energy (unfortunately none helps to solve the vacuum energy problem). The first one is simply an assumption 
that DE is the energy of a slowly varying sterile scalar field $\phi$ with the energy-momentum tensor given by eq. (8). This field is similar to the inflaton but at much lower energy scale. For example the inflaton mass could be as large as $10^{-5} \mathrm{~m}_{P l} \approx 10^{14} \mathrm{GeV}$, while the mass of the contemporary dark energy field $\phi$ should be smaller than the Hubble parameter today, so the field remains frozen during the Hubble time. When at a distant future the Hubble parameter becomes smaller than $m_{\phi}$ or the curvature of the potential $U(\phi)$, the universe acceleration would turn back to deceleration. The equation of state of $\phi$ would be either nonrelativistic one with $P=0$ if $m_{\phi} \neq 0$, or the relativistic with $P=\rho_{\phi} / 3$, if the potential has the form $U(\phi)=\lambda \phi^{4}$. Correspondingly we come back to the usual Friedman cosmology with one-to-one correspondence between geometry and the final destiny of the universe: eternal expansion or big crunch. Here lies an essential difference between Lambda-term and the energy of dynamical scalar field.

Another possibility to describe accelerated cosmological expansion is to assume that gravitational action is modified at low curvature by an addition of a nonlinear function of the curvature scalar $F(R)$ :

$$
S=\frac{m_{P l}^{2}}{16 \pi} \int d^{4} x \sqrt{-g}[R+F(R)]+S_{m} .
$$

Here the first term, proportional to $R$, is the usual Hilbert-Einstein action of GR, and $S_{m}$ is the action of matter fields. The function $F(R)$ is constructed in such a way that in absence of matter the gravitational field equations have the De Sitter like solution, $R=$ const, which describes an accelerated cosmological expansion.

A nonlinear function of $R$ in the action leads to higher order equations of motion, so care should be taken to avoid ghosts, tachyons, unstable solutions strongly deviating from the GR, and the solutions leading to gravitational singularities. There are several similar types of $F(R)$ satisfying these conditions [50]. For example, an explicit form $F(R)$ suggested in the first paper in this reference is:

$$
F(R)=\lambda R_{0}\left[\left(1+\frac{R^{2}}{R_{0}^{2}}\right)^{-n}-1\right]-\frac{R^{2}}{6 m^{2}},
$$

where the last term is added to prevent from past singularity in cosmology [50] and future singularity in systems with rising energy/mass density [51], $R_{0}$ is a constant parameter close by magnitude to the cosmological curvature in the contemporary universe, $n$ is an integer, $\lambda=$ const is dimensionless, and $m=$ const is a parameter with dimension of mass.

The modified gravity theories possess some peculiar features which may either lead to restrictions of the values of the parameters or to very interesting predictions which can be verified by observations. In contracting astronomical systems with rising mass density high frequency oscillations of $R$ with the amplitude much larger than the canonical GR value [52] are induced. The oscillating $R$ effectively produces elementary particles which may make noticeable contribution to high energy cosmic rays.

Another striking effect of $F(R)$ theories is gravitational repulsion inside collapsing matter clouds [53], which might lead to formation of cosmic voids.

\subsection{Cosmological antimatter}

According to the simplest scenarios of baryogenesis there is a universal excess of matter over antimatter in all the universe, so the baryon asymmetry, $\beta=\left(n_{B}-n_{\bar{B}}\right) / n_{\gamma}$ has the same magnitude and sign everywhere. According to this picture there is no room for primordial cosmic antimatter. Observed antiprotons and positrons are supposed to be of secondary origin. The observed flux of antiprotons agrees with theoretical expectations but there are some anomalies in positrons whose origin is not understood. First, there is a powerful $0.511 \mathrm{MeV}$ gamma ray line emitted in the Galactic center [54], 
which surely comes from $e^{+} e^{-}$-annihilation at rest. Presumably the annihilation proceeds in positronium, since the probability of its formation is quite high if low energy positrons are present in the interstellar medium. Several possible mechanisms for the origin of positrons are considered in the literature. It is mostly assumed that the low energy positrons are created one way or another in the galactic center at the present time, see the paper [55] and references therein. However the positrons may be primordial, as well, appearing from some primordial antimatter objects [56].

Recently an unexpectedly high flux of energetic positrons in cosmic rays was discovered by PAMELA [57] and confirmed by two other groups [58, 59]. However, these positrons are surely of secondary, but mysterious, origin. The observed spectrum of these energetic positrons and the band of theoretical expectations for their flux, according to the work [62], are presented in fig. 3.

In the recent years primordial antimatter was actively searched for in several detectors [57, 59, 61] looking for anti-helium in cosmic rays. The production of antinuclei in secondary processes is strongly suppressed. E.g. antideuterium can be produced in collisions of cosmic antiprotons with interstellar hydrogen or helium. First an antineutron can be produced in this reaction and then it may be captured by proton in interstellar gas. According to the estimates of ref. [60] the flux of antideuterium, created by this sequence of reactions, would be at the level $10^{-7} / \mathrm{m}^{2} / \mathrm{s} / \mathrm{GeV} / \mathrm{str}$. This flux is nine orders of magnitude below the observed flux of ${ }^{4} \mathrm{He}$. Evidently the secondary production of heavier nuclei should be much weaker. As estimated in the same paper [60], the flux of the secondary produced antihelium- 3 and antihelium- 4 should be smaller by 4 and 8 orders of magnitude respectively. So a registration even a single anti-helium nucleus would be a strong indication on existence of primordial antimatter. At the moment only an upper bound on the flux of antihelium-4 is known which is 8-9 orders of magnitude below the helium-4 flux [57, 59, 61].

If primordial antinuclei are observed, it would mean that whole antiworlds: antigalaxies, antistars, antiplanets, even antipeople may exist. In this connection it is very interesting to know if and how it could possible to distinguish an antistar from a normal star distantly by astronomical observations. It may be feasible for stars which are not too far away i.e. for those in the Galaxy. A model of abundant antistellar population in the Galaxy was suggested in papers [63] and its phenomenological implications are studied in $[56,64]$, in particular, the observational signature of antistars.

It would be exciting if the foreseeing by Arthur Shuster who conjectured that that there could be entire solar systems, made of antimatter and indistinguishable from ours [65] and by Paul Dirac: "It is quite possible that... for some of the stars it is the other way about, these stars being built up mainly of positrons and negative protons. In fact, there may be half the stars of each kind. The two kinds of stars would both show exactly the same spectra, and there would be no way of distinguishing them by present astronomical methods... Maybe there exists a completely new universe made of antimatter?" [66], happen to be true. However, as we now the spectra and properties of emitted particles from stars and antistars may be different due to CP-violation. So probably Schuster and Dirac were too pessimistic about this point.

\section{Problems}

Because of lack of time and space, we present in this section only a list of unsolved cosmological and astrophysical problems, mysteries, and non yet answered questions. The list is surely not complete and reflects the author's personal preferences. It inlcludes:

1. What makes cosmological dark matter? Elementary particles or microscopically large objects? What kind of particles or objects explicitly?

2. What is the mechanism of cosmological acceleration: dark energy (and in particular vacuum energy) or modified gravity, or something else? 


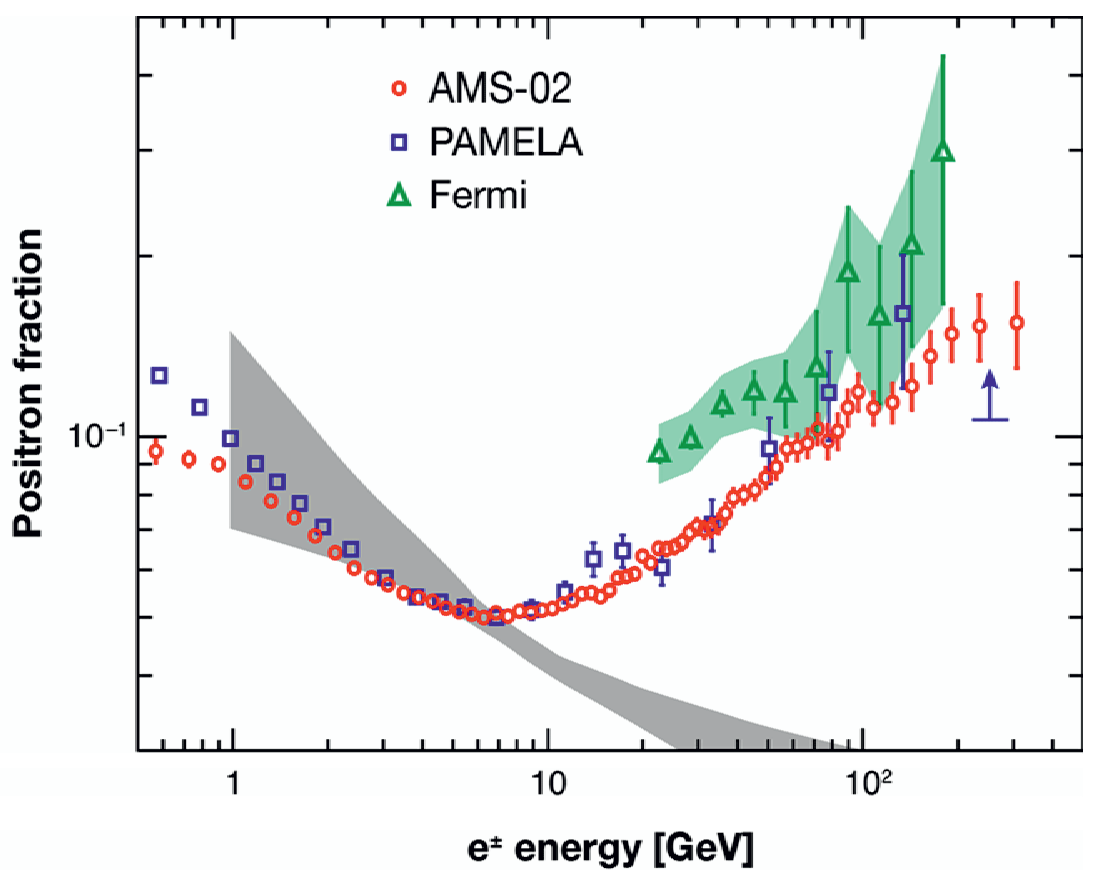

Figure 3. Spectrum of cosmic ray positrons according to measurements of PAMELA, Fermi, and AMS, as presented in ref. [59]. Grey area indicates theoretical expectations for the positron flux according to ref. [62]. 
3. How one can explain the cosmic conspiracy according to which completely different forms of matter/energy (baryonic matter, dark matter, and dark energy) have comparable magnitudes, $\Omega_{b} \sim \Omega_{D M} \sim \Omega_{D E}$ ?

4. What out of many mechanisms of baryogenesis is realized in nature? Or do there exist several scenarios with comparable efficiency?

5. Is there primordial cosmic antimatter? Are there antistars in the universe and in our Galaxy?

6. What is the origin of $0.511 \mathrm{MeV} e^{+} e^{-}-$annihilation line from the Galactic center? Are there similar, though less intensive, "annihilators" in the galactic halo?

7. What is the mechanism of the excessive production of high energy positrons discovered by PAMELA and confirmed by Fermi and, especially, by AMS?

8. What is the origin of ultra high energy cosmic rays (UHECR) up to $10^{21} \mathrm{eV}$ and how the shape of their spectrum can be explained?

9. How superheavy black holes, observed in the centers of all(?) large galaxies were created? What was created first: galaxies and then black holes inside these galaxies or, vice versa, first black holes which seeded the galaxy formation? If the high redshift quasars are such black holes? Why the matter around these early formed quasars is enriched by metals, which normally appear as a result of supernova explosions for which some enduring prehistory is necessary?

10. Which is the mechanism of gamma-burster radiation, especially those seen at high $z$ ?

11. What is the origin of large scale galactic and intergalactic magnetic fields?

12. Do low multipole anomalies in $\mathrm{CMB}$ point to a large scale cosmological inhomogeneity/unisotropy of the universe?

13. How serious is the discrepancy between theory and observations for the abundance of ${ }^{7} \mathrm{Li}$ ? Does it indicate a new physics operating at BBN epoch or there is a problem with interpretation of the observational data?

14. What is the nature of dark radiation which existence is possibly indicated by the analysis of BBN and CMB? Could this radiation consist of sterile neutrinos, mirror matter or none of the above? Is this radiation real or an observational artifact?

15. Last but not least: which mechanism almost eliminated huge and experimentally known contributions to vacuum energy with 45 orders of magnitude precision? This seems to be the central problem not only in cosmology but in whole fundamental physics.

\section{Perspectives}

With new instruments, both ground based and on-satellite, working in all bands of electromagnetic radiation, with gravitational wave interferometers, neutrino telescopes, and cosmic ray detectors great progress in understanding of the universe is expected. Surely some but, most probably, not all the problems enumerated in the previous section will be solved. On the other hand, new instruments will surely reveal new mysteries in the sky demanding new physics beyond our present day knowledge and demanding new instruments for their understanding.

Acknowledgement. I acknowledge the support by the grant of the Russian Federation government 11.G34.31.0047.

\section{References}

[1] A. Einstein, Preuss. Akad. der Wissenschaften, Sitzung., 1915 (part 2), 844; Ann. der Physik 49, 769 (1916). 
[2] A. Friedmann, Z. Phys. 10, 377 (1922); Z. Phys. 21, 326 (1924).

[3] G. Lemaitre, Annales de la Socété scientifique de Bruxelles, Série A, 47, 49 (1927); English translation in MNRAS, 91, 483 (1931); see also H. Nussbaumer, L. Bieri, arXiv:1107.2281v2 [physics.hist-ph].

[4] E. Hubble, PNAS, 15, 168 (1929).

[5] G. Gamow, Phys. Rev. 70 (1946) 572;

R. A. Alpher, H. A. Bethe, G. Gamow, Phys. Rev. 73, 803 (1948);

G. Gamow, Phys. Rev. 74, 505 (1948). R. A. Alpher, R. C. Herman, Nature 162, 774 (1948);

R. A. Alpher, R. C. Herman, G. Gamow, Phys. Rev. 74, 1198 (1948).

[6] A.A. Penzias, R. Wilson, Astrophys. J. P142, 419 (1965).

[7] Ya. B. Zeldovich, Adv. Astron. Ap. 3, 42 (1965).

[8] Ya. B. Zeldovich, L.B. Okun, S.B. Pikelner, Uspekhi Fiz. Nauk 87, 113 (1965)

[9] B.W. Lee, S. Weinberg, Phys. Rev. Lett. 39, 165 (1977).

[10] M.I. Vysotsky, A.D. Dolgov, Ya.B. Zelodvich, ZhETF Letters, 26, 200 (1977).

[11] I.Yu. Kobzarev, L.B. Okun, I.Ya. Pomeranchuk, Yadernaya Fizika (Soviet Journal of Nuclear Physics), 3, 1154 (1966).

[12] S.S. Gerstein, Ya.B. Zeldovich, Pis'ma ZhETF, 4, 174 (1966).

[13] R. Cowsik, J. McClelland, Phys. Rev. Lett. 29, 669 (1972).

[14] A.D. Dolgov, Phys. Repts. 370, 333 (2002).

[15] A. D. Sakharov, Pis'ma ZhETF, 5, 32 (1967).

[16] A.A. Penzias, R. Wilson, Astorphys. J. 142, 419 (1965).

[17] A.G. Doroshkevich, I.D. Novikov, DAN USSR, 154, 809 (1964).

[18] T.A. Ter Shmaonov, Pribory i Tekhnika Ekspreimenta (Devicies and technique of Experiment) 1 (1957) 83.

[19] J. H. Oort, Bull. Astron. Inst. Netherlands, 6, 249 (1932).

[20] F. Zwicky, Helv. Phys. Acta, 6, 10 (1933).

[21] J. Einasto, A. Kaasik, E. Saar, Nature 250, 309 (1974).

[22] J. P. Ostriker, P. J. E. Peebles, A. Yahil, Astrophys. J., 193. L1 (1974).

[23] G.F. Smoot et al, Astrophys. J. 396, L1 (1992).

[24] I.A. Strukov, A.A. Bryukhanov, D.P. Skulachev, M.V. Sazhin, Astron. J. Lett. 18, 387 (1992), 387.

[25] Planck Collaboration: P. A. R. Ade, et al, arXiv:1303.5076 [astro-ph.CO].

[26] A.F. Heckler, Phys. Rev. D bf 49, 611 (1994);

R.E. Lopez, S. Dodelson, A. Heckler and M.S. Turner, Phys. Rev. Lett. 82, 3952 (1999).

[27] A.D. Dolgov, M. Fukugita, JETP Lett. 56, 123 (1992); Phys. Rev. D 46, 5378 (1992).

[28] A.D. Dolgov, S.H. Hansen and D.V. Semikoz, Nucl. Phys. B 503 (1997) 426; Nucl. Phys. B 543 (1999) 269.

[29] A.D. Dolgov, Phys. Repts. 370, 333 (2002).

[30] G. Mangano, G. Miele, S. Pastor, M. Peloso, Phys.Lett., B 534, 8 (2002)

[31] Y. I. Izotov, G. Stasinska, N. G. Guseva, arXiv:1308.2100 [astro-ph.CO].

[32] R. Cooke, M. Pettini, R.A. Jorgenson, M.T. Murphy, Ch.C. Steidel, arXiv:1308.3240 [astroph.CO]

[33] D. Kazanas, Astrophys. J. 241, L59 (1980).

[34] A. Guth, Phys. Rev. D 23347 (1981).

[35] A. D. Linde, Phys. Lett. B 129, 177 (1983). 
[36] A.A. Starobinsky, Phys. Lett. B 91, 99 (1980).

[37] V.F. Mukhanov, G.V. Chibisov, JETP Lett. ‘ 33, 532 (1981); [ Pisma Zh. Eksp. Teor. Fiz. 33, 549 (1981)].

[38] E.R. Harrison, Phys. Rev. D 1, 2726 (1970); Ya.B. Zeldovich, MNRAS. 160, 1 (1972).

[39] A.A. Starobinsky, Pisma Zh. Eksp. Teor. Fiz. 30, 719 (1979);

V.A. Rubakov, M.V. Sazhin, A.V. Veryaskin, Phys. Lett. B 115, 189 (1982).

[40] A.D. Dolgov, P.D. Naselsky, I.D. Novikov, astro-ph/0009407;

A.D. Dolgov, D. Ejlli, Phys. Rev. D 84, 024028 (2011).

[41] A.D. Dolgov, Phys. Rep. 222, 309 (1982).

[42] B.D. Fields, Annual Review of Nuclear and Particle Science, 61, 47 (2011).

[43] T. Kaluza, Sitzungsber. Preuss. Akad. Wiss. Berlin (Math. Phys.) 966 (1921);

O. Klein, Z. Phys. A 37, 895 (1926).

[44] V.A. Rubakov, M.E. Shaposhnikov, Phys. Lett. B, 125, 136 (1983).

[45] Z.G. Berezhiani, L. Kaufmann, P. Panci, et al CERN-PH-TH-2008-108;

Z. Berezhiani, Int. J. Mod. Phys. A 19, 3775 (2004);

P. Ciarcelluti, Int. J. Mod. Phys. D 19, 2151 (2010);

R. Foot, e-Print: arXiv:1307.1755 [astro-ph.GA].

[46] E. Krylov, A. Levin, V. Rubakov, Phys.Rev. D 87, 083528 (2013) and references therein.

[47] K. Lawson, A.R. Zhitnitsky, e-Print: arXiv:1305.6318 [astro-ph.CO].

[48] M. Gell Mann, R.J. Oakes, B. Renner, Phys. Rev. 175, 2195 (1968).

[49] M.A. Shifman, A.I. Vainshtein, V.I. Zakharov, Nucl. Phys. B 147, 385 (1978).

[50] A.A. Starobinsky, JETP Lett. 86, 157 (2007);

W. Hu, I. Sawicki, Phys. Rev. D 76, 064004 (2007);

A. Appleby, R. Battye, Phys. Lett. B 654,6 (2007).

[51] A. V. Frolov, Phys. Rev. Lett. 101, 061103 (2008);

E. V. Arbuzova, A. D. Dolgov, Phys. Lett. B 700, 289 (2011).

[52] E.V. Arbuzova, A.D. Dolgov, L. Reverberi, Eur. Phys. J. C 72, 2247 (2012);

E.V. Arbuzova, A.D. Dolgov, L. Reverberi, Phys. Rev. D 88, 024035 (2013).

[53] E.V. Arbuzova, A.D. Dolgov, L. Reverberi, Astroparticle Physics 54C, 44 (2014); arXiv:1306.5694.

[54] W.N. Johnson, F.R. Harnden, R.C. Haymes, Astrophys. J. 172, L1 (1972);

M. Leventhal, C.J. MacCallum, P.D. Stang, Astrophys. J. 225, L11 (1978);

W.R. Purcell et al. Astrophys. J. 491, 725 (1997);

P.A. Milne, J.D. Kurfess, R.L. Kinzer, M.D. Leising, New Astron. Rev. 46, 553 (2002);

J. Knodlseder et al., Astron. Astrophys. 441, 513 (2005);

P. Jean et al., Astron. Astrophys. 445, 579 (2006);

G. Weidenspointner et al., Astron. Astrophys. 450, 1013 (2006).

[55] C. Bambi, A.D. Dolgov, A.A. Petrov, Phys. Lett. B 670, 670 (2008); 681, 504 (2009), Erratum.

[56] C. Bambi, A.D. Dolgov, Nucl. Phys. B 784, 132 (2007).

[57] O. Adriani, G. C. Barbarino, G. A. Bazilevskaya et al., Nature, 458, 607 (2009);

Mikhailov, V.V. et al. Bull. Russ. Acad. Sci. Phys. 77, 1309 (2013).

[58] M. Ackermann et al., Phys. Rev. Lett. 108, 011103 (2012).

[59] AMS Collaboration (M. Aguilar et al.), Phys. Rev. Lett. 110, 141102 (2013).

[60] R. Duperray, B. Baret, D. Maurin, G. Boudou, A. Barrau, L. Derome, K. Protasov, M. Buenerd,

Phys. Rev. D 71083013 (2005). 
[61] K. Abe, H. Fuke, S. Haino S, et al, BESS Collaboration, Phys. Rev. Lett. 108, 131301 (2012).

[62] I. V. Moskalenko, A. W. Strong, J. F. Ormes, M. S. Potgieter, Astrophys. J. 565, 280 (2002).

[63] A. Dolgov, J. Silk, Phys. Rev. D 47, 4244 (1993);

A.D. Dolgov, M. Kawasaki, N. Kevlishvili, Nucl. Phys. B 807, 229 (2009).

[64] A.D. Dolgov, I.B. Khriplovich, A.S. Rudenko, Pis'ma ZhETF (JETP Lett.), 96, 241 (2012);

A.D. Dolgov, V.A. Novikov, M.I. Vysotsky, Pis'ma ZhETF (JETP Lett.), 98, 587 (2013);

A.D. Dolgov, S.I. Blinnikov, arXiv:1309.3395 [astro-ph.CO], Phys. Rev. D (to be published). [65] A. Schuster, Nature, 58, 367 (1898).

[66] P. Dirac, Nobel Lecture, December 12, 1933, Theory of electrons and positrons. 